\title{
Clubs de lectura en la universidad: mirando a la biblioteca pública'
}

\author{
University Reading Clubs: Looking at the Public Library
}

\author{
MARÍA ANTONIA MORENO-MULAS \\ ARACELI GARCÍA-RODRÍGUEZ \\ RAQUEL GOMEZ-DÍAZ \\ Universidad de Salamanca \\ España \\ morenomulas@usal.es \\ araceli@usal.es \\ rgomez@usal.es
}

(Recibido: 24/II/2OI8; aceptado: 28/04/2OI9)

Resumen. La biblioteca pública en España en su labor de promoción lectora ha apostado por los clubs de lectura, tanto en su modalidad presencial como, en los últimos años, virtual o en la nube. Los clubs se han revelado, además, herramientas eficaces para cohesionar a grupos de personas a través de la socialización de la lectura. ¿Se ha de mirar a la biblioteca pública y a sus clubs, para adaptarlos al ámbito universitario?

A través del análisis de una selección de experiencias desarrolladas en bibliotecas públicas y universitarias españolas, entre las que se incluye la puesta en marcha de dos clubs en el ámbito universitario enmarcados en el Proyecto de Investigación "Los clubs de lectura como herramienta para la promoción lectora en el ámbito académico y escolar" (Fundación Solórzano Barruso FS/S-2017) de la Universidad de Salamanca, se llega a la conclusión de que para que un club de lectura virtual funcione, es necesaria una planificación rigurosa, una adecuada elección de la red social que se vaya a utilizar, la formación previa del coordinador y los lectores, la dinamización de la conversación y la integración de experiencias presenciales en el club virtual.

Palabras clave: club literario; promoción de la lectura; bibliotecas universitarias; bibliotecas públicas; universidad.
Abstract. The public library in Spain, in its effort towards reading promotion, has opted for book clubs, both in a face-to-face mode and, as in recent years, virtually or within the cloud. The clubs have proved to be, additionally, as effective tools to constructs groups of people through the socialization of reading. Should we take a look at the public library and its clubs, and adapt them to the university environment? Through the analysis of a selection of experiences developed within Spanish public and university libraries, including the setting up of two clubs in a university context framed by the Research Project "Book clubs as a tool for reading promotion in the academic and school environment" (Solórzano Barruso Foundation FS / S-2017) from the University of Salamanca, it is concluded that for a virtual reading club to work it is necessary to plan rigorously, to choose an appropriate social network that will be utilized, to previously train the coordinator and the readers, to energize conversations and to integrate face-to-face experiences in the virtual club.

Keywords: book club; reading promotion; academic libraries; public libraries; university.

\footnotetext{
I Para citar este artículo: Moreno-Mulas, Ma Antonia; García-Rodríguez, Araceli y Gómez-Díaz, Raquel (2O2O). Clubs de lectura en la universidad: mirando a la biblioteca pública. Alabe 21. [www.revistaalabe.com]

DOI: IO.I5645/Alabe2O2O.2I.5
} 


\section{Los clubs de lectura: herramientas eficaces de promoción de la lectura no acadé- mica}

Un club de lectura está formado por grupos de personas que leen a la vez (de manera individual y sin un espacio predeterminado), un libro pactado previamente y se reúnen, con cierta periodicidad, para conversar sobre él. Los clubs en España han experimentado un crecimiento exponencial (Arana, 2OI7), amparados en muchos casos por la biblioteca pública que, en su labor de promoción de la lectura, ha apostado firmemente por la implementación de clubs presenciales, virtuales a través de redes sociales o plataformas específicas (García-Rodríguez, y Gómez Díaz, 20I9 p. I76-I78) y/o en la nube (Moreno-Mulas, García-Rodríguez, Gómez-Díaz, 20I7). Estos clubs, además, se han revelado como herramientas eficaces para cohesionar a personas en grupos donde descubren al otro, lo diferente, a través de la socialización de la lectura. Las obras que se leen en el seno de los clubs comprenden un amplio arco temático: novelas que ponen el acento en lo ecológico, las econovelas; el teatro; la literatura infantil; la poesía; la novela histórica o la novela negra. Junto a éstos, coexisten los clubs generalistas que buscan apartar a sus miembros de la zona de confort, esto es, de sus gustos habituales de lectura. No hay que olvidar la pujanza de leer en otras lenguas distintas a la materna; cada vez es más habitual comentar lecturas en versión original que ayudan a enriquecer el vocabulario, la expresión escrita y la expresión oral. Sin embargo, la característica principal son los lectores que los forman y quiénes determinarán, por lo tanto, la selección de lecturas y su dinamización: niños, jóvenes, adultos, familiares, o intergeneracionales.

Otro aspecto importante a destacar es que los clubs de lectura organizados desde la biblioteca pública suelen convertirse en un importante activo dentro de la actividad de la biblioteca y de su comunidad; sus miembros participan de una manera muy activa colaborando en la organización, desarrollo y difusión de programas y actos (Moreno-Mulas, et.al., 2OI7). A la par, estos lectores se convierten en objetivos prioritarios para las bibliotecas públicas en materia de formación en distintos ámbitos, especialmente en lectura digital y lectura social.

Pero, ¿qué sucede en el ámbito universitario cuando hablamos de lectura no académica? ¿Dónde está la clave para que estas prácticas lectoras en las bibliotecas públicas no pierdan fuerza con el paso del tiempo, sino todo lo contrario? ¿Desde la biblioteca universitaria puede copiarse/pegarse el modelo de club virtual impulsado por la biblioteca pública o, por el contrario, hay que establecer cuáles son los más adecuados para cada ámbito?

Para responder a estas preguntas, llevamos la experiencia de la biblioteca pública a la universidad organizando dos clubs de lectura virtuales entre los alumnos del Grado en Información y Documentación de la Universidad de Salamanca, enmarcados en el Proyecto de Investigación "Los clubs de lectura como herramienta para la promoción lectora en el ámbito académico" de la (Fundación Solórzano Barruso FS/S-2OI7) 


\section{Objetivos}

Considerando que los clubs de lectura puestos en marcha desde las bibliotecas públicas, en cualquiera de sus modalidades, se han revelado como herramientas eficaces para la promoción de la lectura, establecimos que era necesario:

Definir algunas de sus claves extrapolables al fomento de la lectura no académica en el ámbito universitario, indicando sus elementos más importantes de cara a su implementación en la universidad.

Definir el modelo más idóneo a la hora de diseñar, organizar, implementar y dinamizar un club de lectura virtual en la universidad.

Buscar la integración de dinámicas presenciales en el seno de un club de lectura virtual para comprobar cómo pueden favorecer la cohesión grupal y la participación de los lectores en la conversación.

\section{Metodología}

Para conseguir los objetivos enumerados se realizó una selección de clubs de lectura con características virtuales; esto es, aquellos en los que la mayoría de las reuniones y el peso de la conversación se realizan en un entorno digital, a través de una red social, plataforma, blog o canal en YouTube.

Previo al diseño y ejecución de dos clubs de lectura virtuales en el ámbito universitario, se analizó una selección de experiencias, con el objetivo de recopilar ideas. Las características y conclusiones, tanto de estas experiencias como de los clubs de lectura virtuales de diseño y realización propios, se exponen a continuación:

\section{a) CLUBIT. Club de lectura juvenil on line de la Biblioteca para Jóvenes Cubit}

Zaragoza. Se trata de un club de lectura que se promociona a través de Instagram y Twitter (@Clubit_ZGZ), con participación de dos jóvenes booktubers y cuya gestión (también de las redes sociales) corre a cargo de la biblioteca. Las obras que se leen en este club pertenecen al género juvenil, y cada mes, se lee una diferente ${ }^{2}$. Al finalizar la lectura de cada obra, los lectores y las booktubers Andreo Rowling (la escritora Andrea Izquierdo) y Dostip Julia, se encuentran en una reunión final en el espacio físico de la biblioteca. Los aspectos más destacables y positivos son la inscripción a través de formularios en línea; la gestión de contenidos en las redes sociales por parte de la biblioteca; la apuesta decidida por Instagram, la red preferida de los más jóvenes y, sobre todo, la alianza con las jóvenes booktubers; una unión natural que se alimenta del Campus Booktuber de la biblioteca y que se encuentra en su tercera edición.

\footnotetext{
${ }^{2}$ Algunos de los títulos leídos en los últimos meses son: Valkiria. Game Over, de David Lozano; Mi otra mitad, de Andrea Izquierdo; La chica invisible de Blue Jeans y Temblor de Maggie Stiefvater.
} 
b) Nubeteca. Plataforma de los clubes de lectura de las bibliotecas de la Diputación de Badajoz y la Fundación Germán Sánchez Ruipérez. Nubeteca (https://nubeteca.odilotk.es/) se concibe como una plataforma en la que conversar y leer en la nube, por lo tanto, la lectura se encuentra accesible a través del propio club y estaríamos hablando de un club de lectura en la nube. Para participar se precisa ser socio de las bibliotecas pacenses que forman parte de esta experiencia, e inscribirse a través de ellas: Don Benito, Fuente del Maestre, Llerena, Montijo y Villafranca de los Barros. Como puntos fuertes destacamos el formato de lectura y conversación en un mismo espacio; la conversación entre lectores, bibliotecarios y autores de diferentes lugares que propicia el acercamiento a la cultura en el mundo rural; el carácter lúdico de la dinamización y la búsqueda del encuentro presencial final (viajes literarios).

c) Descubre tu lectura. Canal de YouTube de las Bibliotecas de los Centros Cívicos de Burgos. Las Bibliotecas de los Centros Cívicos de Burgos unen sus clubs y los muestran al resto del mundo a través de su canal en YouTube Descubre Tu Lectura (bit.ly/descubretulectura). Destacamos la virtualidad que se cuela en lo presencial a través de los booktrailers realizados por los propios bibliotecarios; las votaciones para elegir lecturas; las entrevistas y/o los vídeos en los que los autores explican las obras; la retransmisión en directo de los coloquios.

d) La calma lectora. Blog del Club de lectura de la Universidad de Las Palmas de Gran Canaria. Desde el curso 2009-20IO, la Universidad de las Palmas de Gran Canaria administra La calma lectora, el blog de su club de lectura (http:/ / bibwp.ulpgc.es/clubdelecturaulpgc/). Entre los aspectos que distinguen este club están los juegos de pistas para anunciar las lecturas; la completa información que se ofrece sobre el autor y la obra; el encuentro presencial final para el que es preciso la inscripción en línea y con número máximo de participantes (20), buscando la conversación y huyendo del acto académico; y la apertura del blog y de las tertulias presenciales a todas las personas interesadas, sin requisito de pertenencia a la comunidad universitaria. 


\section{Dos clubs de lectura virtuales en la Universidad}

Game Overy Somosun@s monstru@s son los dos clubs de lectura desarrollados mayoritariamente de forma virtual, formados por alumnos del Grado de Información y Documentación de la Universidad de Salamanca, y enmarcados en el Proyecto de Investigación "Los clubs de lectura como herramienta para la promoción lectora en el ámbito académico" de la (Fundación Solórzano Barruso FS/S-2OI7). Ambos clubs se realizaron en grupos cerrados de Facebook y la participación, voluntaria, desde el mismo momento de la inscripción, pasando por la asistencia a algunas actividades presenciales.

Para articular la conversación y la dinamización de las obras Valkiria. Game Over, de David Lozano y No soy un monstruo de Carmen Chaparro, se procedió a una lectura analítica en la que se desbrozaron las tramas principales, las secundarias o subtramas; los contextos temporales, geográficos; los personajes y sus motivaciones (Figura i); así como los temas que son susceptibles de ser tratados en profundidad y recursos para enriquecer la conversación; recursos que apuntalan estos temas. Este análisis se concreta en una deconstrucción de las obras y, sobre su base, se diseñan los proyectos de dinamización de los clubs virtuales, esto es, la conversación (Tabla I). Estos materiales de trabajo se presentaron, a lo largo de 20I8, en el III Congreso Internacional Educación Socialy Alfabetizaciones. (Eco)Sistemas de la lectura: de la alfabetización a la educación. Cuenca, 7 de junio de 2oI8; el VII Congreso Internacional de la SEMYR, Patrimonio textualy Humanidades digitales. Universidad de Salamanca, 4-6 de septiembre de $208^{3}$.

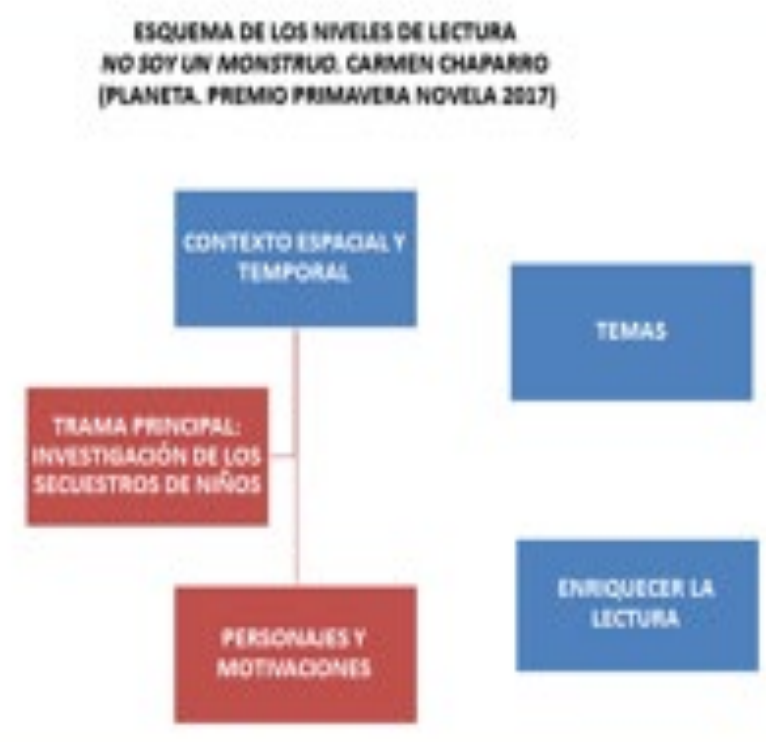

3 En prensa. 


\begin{tabular}{|c|c|}
\hline TEMA & ENRIQUECIMIENTO \\
\hline $\begin{array}{l}\text { David } \\
\text { Lozano }\end{array}$ & $\begin{array}{l}\text { https://es.literaturasm.com/autor/david-lozano-garbala } \\
\text { http://www.twitter.com/DavidlozanoG } \\
\text { https://www.instagram.com/davidlozanog/ } \\
\text { https://www.facebook.com/david.lozanogarbala }\end{array}$ \\
\hline $\begin{array}{c}\text { Valkiria: } \\
\text { Game Over (SM) }\end{array}$ & $\begin{array}{l}\text { Así se hizo el unboxing del que todo el mundo habla } \\
\text { https://es.literaturasm.com/actualidad/valkiria-asi-hizo-unboxing-que- } \\
\text { todo-mundo-habla } \\
\text { David Lozano presenta Valkiria: Game Over } \\
\text { https://youtu.be/neL5nFXt4Sc }\end{array}$ \\
\hline Tor & La red Tor https://www.youtube.com/watch?v=BGW6TMwZsnQ \\
\hline $\begin{array}{l}\text { Redes sociales, } \\
\text { privacidad... }\end{array}$ & $\begin{array}{l}\text { ¿Por qué me vigilan si no soy nadie? Marta Peirano I TEDxMadrid } \\
\text { https://youtu.be/NPE7i8wuupk } \\
\text { El lado oscuro de las redes sociales https://youtu.be/trGgBTbj4z8 } \\
\text { Hacking https://youtu.be/zQ470q7z91k }\end{array}$ \\
\hline Suicidio & $\begin{array}{l}\text { El puente entre el suicidio y la vida } \\
\text { https://www.ted.com/talks/kevin_briggs_the_bridge_between_suici- } \\
\text { de_and_life?language=es }\end{array}$ \\
\hline
\end{tabular}

Tabla 1. Temas y recursos para enriquecer la lectura de Valkiria. Game Over. Elaboración propia

a) Game Over: presencial y virtual. Un binomio que funciona. Desarrollado entre el I6 de abril y el I5 de mayo de 20I8, en él participaron una veintena de alumnos. La novela que se leyó fue Valkiria. Game Over (SM) de David Lozano; se trata de una obra juvenil con tintes de novela negra, protagonizada por jóvenes. La forma de inscripción y captación de los participantes fue en línea pero con interacción presencial. El autor fue cómplice desde el inicio, se le entregaron los materiales internos de trabajo (deconstrucción, proyecto de dinamización y enlaces a recursos) y se concertó un encuentro virtual final en el grupo de Facebook. Es destacable, asimismo las reuniones presenciales con los alumnos antes de comenzar el club en el que se explicaron la forma de participar en el club y la implicación del autor. La conversación en el grupo de Facebook tuvo componentes lúdicos e interactivos, como las fotos de sus mesas de estudio con la novela o la creación de bandas sonoras; y como actividad presencial se llevó a cabo una búsqueda de libros a través de pistas lanzadas en el club, inspiradas en la novela. Los libros, se escondieron en diferentes lugares de la Facultad de Traducción y 
Documentación de la Universidad de Salamanca (lavabos, jardineras, buzones, máquina del café...) e iban convenientemente identificados y envueltos para que su hallazgo fuera, todavía, más sorpresivo.

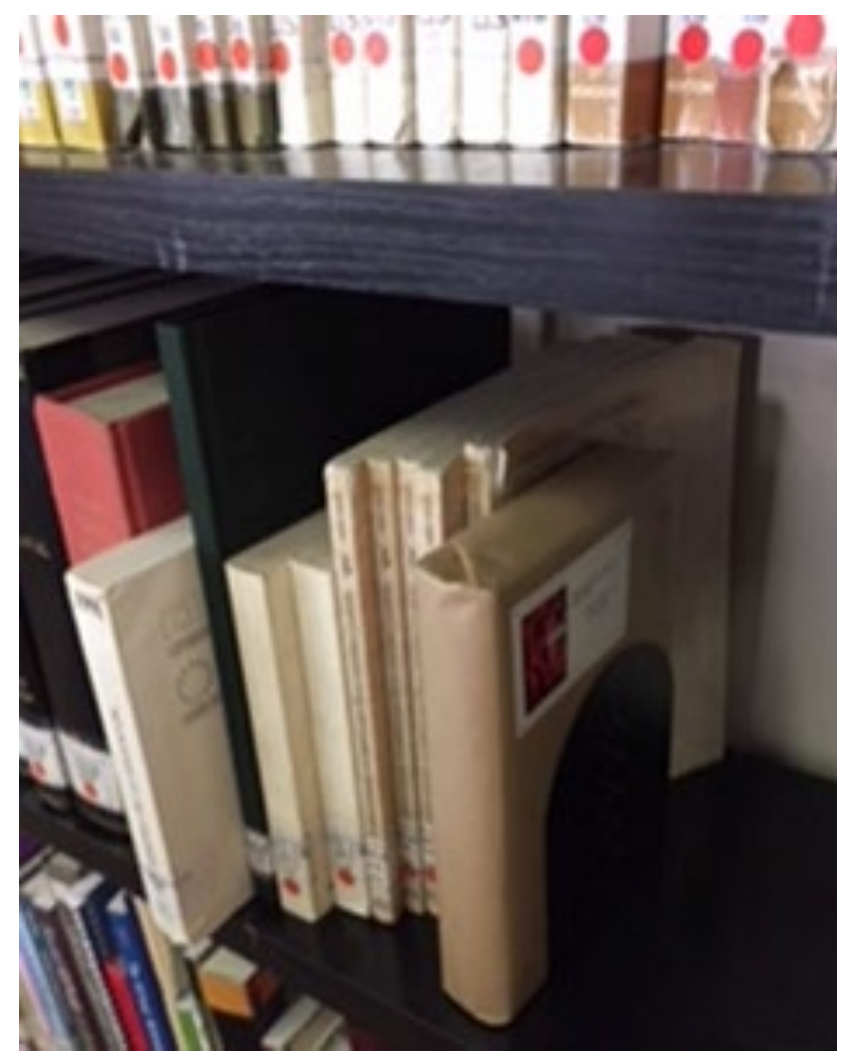

Figura 2. Libros ocultos en distintos lugares de la Facultad de Traducción y

Documentación.

Universidad de Salamanca

Cuando finalizó la experiencia, se dio una charla a los alumnos para explicarles el proceso de organización, implementación y dinamización del club de lectura virtual.

\section{¡Buenos dias y bienvenidos a Game Overt}

(CEste grupo será nuestro lugar de encuentro, nuestro espacio de conversaciōn, đonde se desarrollará nuestro club de lectura en torno a la obra de David Lozano Garbala (SM) "Valkiria: Game Over".

-El club comenzara el próximo lunes 16 de abril, y tendrá una duración de cuatro semanas, hasta el 14 de mayo.

LWEl ritmo de lectura (y de conversación) será el siguiente: cada semana, diez capitulos. Por lo tanto, para el lunes, hablaremos .... Ver más

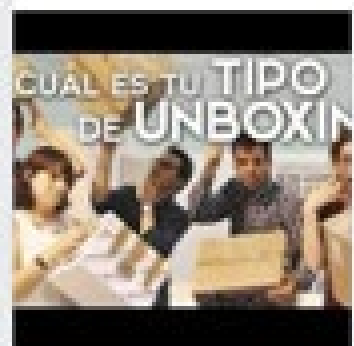

YOUTUEE.COM

TIPOS DE UNBOXING + Valkiria | David

Lozano, Sebas G Mouret, La cueva de Charles, Nerea Marco

Esta semana David Lozano va a abrir la caja de su.

Figura 3. Post de inicio en Facebook del grupo de Game Over. 
A lo largo de la conversación, se abrieron 26 post por parte de la mediadora y i por el autor, que corresponde con su conversación con los lectores. Cada post se acompañaba de enlaces a recursos (documentales, páginas web,) o de propuestas para crearlos: listas de canciones, palabras que definieran sus sensaciones al finalizar la lectura. Estas propuestas creativas constituyeron una excusa perfecta para la reflexión y el debate.

Estos post dieron lugar a un total de 536 comentarios, de los cuales, 262 un $56 \%$ pertenecen a la mediación, 30, un $6 \%$ al autor y el resto, I77, un $38 \%$ a los lectores universitarios.

Los 30 comentarios del autor corresponden, casi en su totalidad (excepto I), a la conversación final. Los comentarios de la mediación, son en su mayoría, respuestas motivadoras a las intervenciones de los alumnos, además de preguntas para incitar a una conversación articulada más allá de acción/reacción.

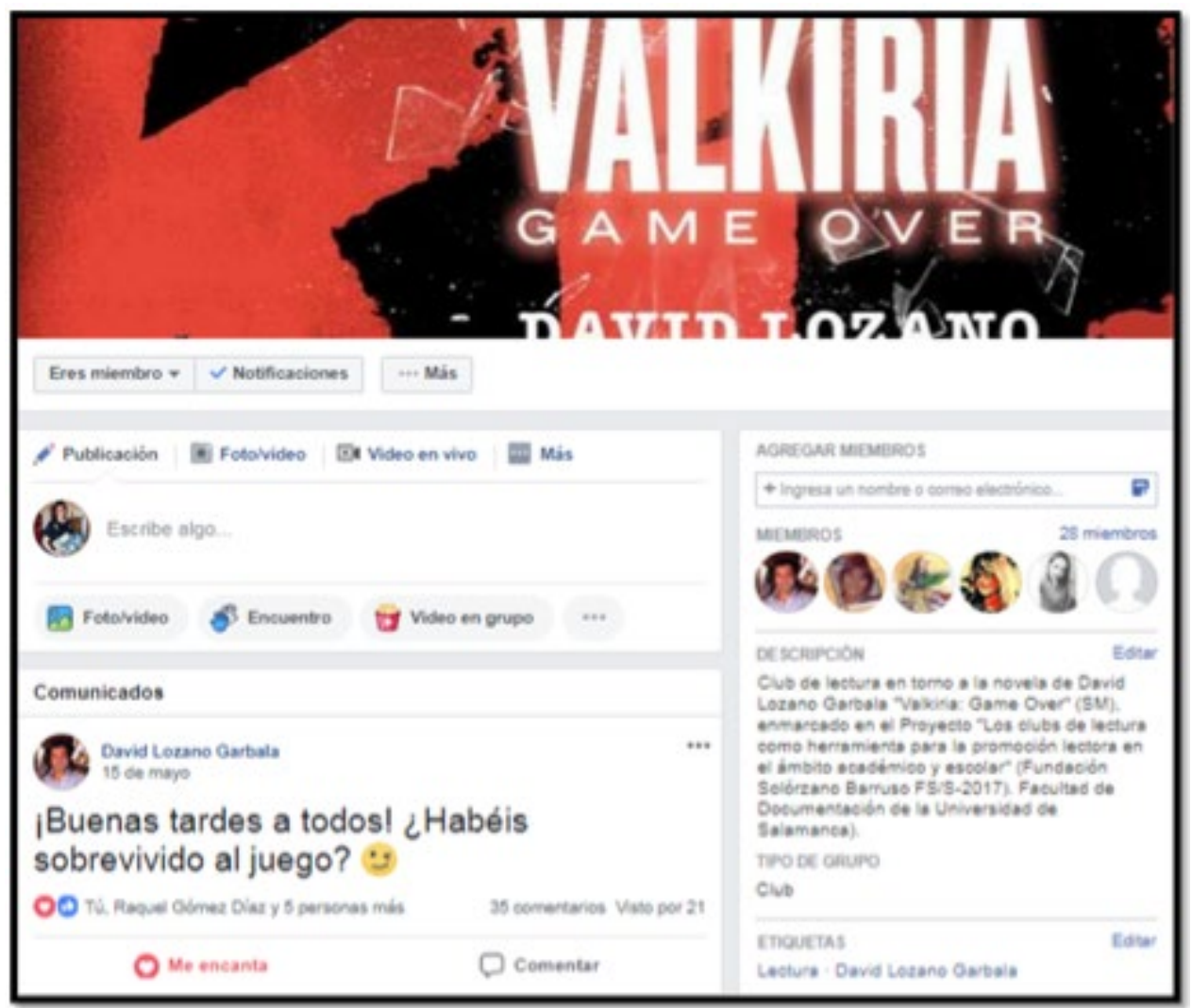

Figura 4. Conversación final con el escritor David Lozano.

Al finalizar la experiencia, se les pasó un cuestionario de satisfacción a los alumnos. Más allá de las opiniones acerca de si gustó la novela más, menos, o nada, los alumnos compartieron reflexiones interesantes que marcan el camino hacia una convivencia entre experiencias virtuales y presenciales, la introducción de procesos creativos y la gamificación. 


\section{Indica qué propuestas del club de lectura te han gustado más}

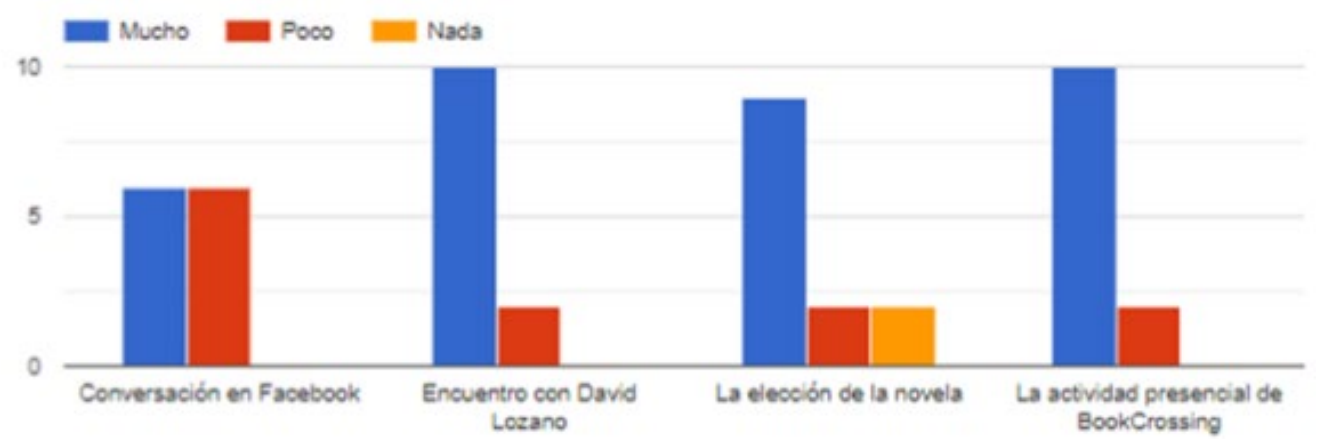

Figura 5. Gráfico de satisfacción de una selección de propuestas

A la pregunta: "una vez que has conocido la experiencia de este club de lectura ¿piensas que en la universidad se deberían organizar este tipo de actividades?", la respuesta fue unánime: Sí, la universidad también es un lugar para la lectura sin fines académicos.

b) Somos un@s monstru@s. Predomina lo virtual sobre lo presencial. Con los resultados explicados, en otoño pusimos en marcha la segunda experiencia, con una novela más adulta (a petición de los participantes). Un thriller, una obra con protagonistas adultos: No soy un monstruo, de Carme Chaparro. Premio Primavera de Novela 201\%. Al club se inscribieron trece alumnos y se realizó entre el 29 de octubre al I6 de noviembre de 20I8, con las siguientes características destacables: las inscripciones se hacen en línea; no se lleva a cabo ninguna sesión presencial para explicar/mostrar el club porque todos, excepto tres, han participado en la experiencia anterior. No se contactó con la autora, se acortó el tiempo de conversación, el toque lúdico fue sensiblemente menor y no se programó la sesión final explicativa. A lo largo de la conversación se abrieron I4 post, cada post se acompañaba de enlaces a recursos (documentales, páginas web, etc.) y de propuestas para conversar: el postureo en las redes sociales, la ética periodística, la profesión de policía o periodista, los avances en neurociencia o los escenarios terroríficos de película o novela. Estos post dieron lugar a un total de 82 comentarios, de los cuales, 62 un $76 \%$ pertenecen a la mediación, y 2O, un $24 \%$ a los lectores universitarios. 


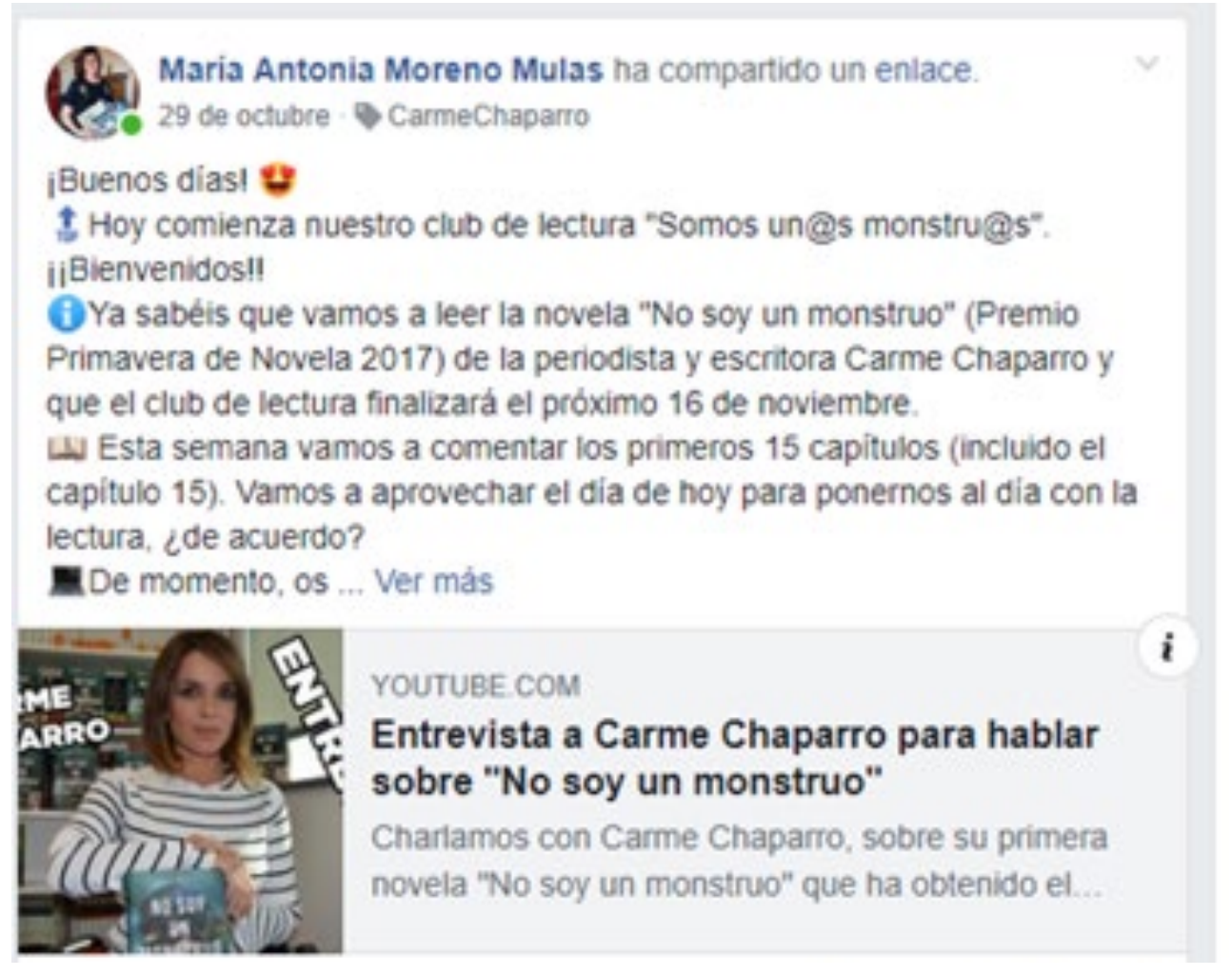

Figura 6. Post de inicio del grupo Somosun@s monstru@s

A la vista de los resultados, nos parece pertinente plantearnos una serie de preguntas. ¿Basta con conocer la experiencia para participar en ella? Parece que no es determinante. ¿Influye la lectura en la participación del club? Pensamos que no, puesto que los lectores nos habían pedido una novela de esas características. ¿Podemos conformarnos, en la organización y desarrollo de un club de lectura virtual, con saber que la mayoría ha leído la novela?

\section{Conclusiones}

Una vez analizadas las distintas experiencias implementadas en la biblioteca pública y tras el contraste con los clubs realizados en la Universidad de Salamanca, hemos constatado que la organización de un club de lectura exige una planificación rigurosa, pero, en los desarrollados en el mundo virtual, se suman una serie de condicionantes entre los que se encuentran la elección del medio donde se realiza, la formación previa de los profesionales, usuarios y autores y el acceso a las obras. Para que un club de estas características funcione, es esencial que exista un coordinador que dinamice la conversación, paute los debates, incorpore elementos multimedia y, sobre todo, sea experto en el uso de las diferentes redes sociales. 
Además, es necesario destacar la importancia de la metodología de análisis previo de la obra. A través de la deconstrucción en tramas principales y secundarias y de la identificación de las motivaciones de los personajes en el marco del contexto histórico, sociopolítico y cronológico, se seleccionan los temas para la conversación y los recursos para el enriquecimiento de la obra

Por otra parte, es esencial la elección de la red social en la que se desarrollará la conversación, teniendo en cuenta la edad de los lectores, las prestaciones, la sencillez en su implementación, la facilidad con la que se pueden incluir comentarios, archivos multimedia, etc.

"Es evidente que las diferencias entre los clubs que realizan reuniones presenciales y los que únicamente funcionan a través de la red influyen en el tipo de interacción que se produce entre los lectores" (Baldaquí, 20I8, p.I38). Por ello, es necesario organizar de forma complementaria clubs virtuales y presenciales y clubs virtuales en los que se incluya algún tipo de actividad que permita el contacto directo y personal de los participantes y, consecuentemente, la socialización, como lo demuestran los datos de participación de los dos clubs puestos en marcha durante el proyecto. En las dos experiencias planteadas se utilizó la misma metodología, pero en la segunda no se propuso ninguna reunión presencial y los datos reflejaron claramente la caída drástica de la participación. El club Game Over supuso un punto de reunión en el que conversar hasta que las reuniones presenciales tuvieran lugar, mientras que en Somos un@s monstru@s, faltó la motivación y el compromiso que la acompaña, poniendo de relieve que una experiencia virtual no es atractiva por sí sola, incluso, en los casos en los que ya se ha participado con anterioridad y poniendo de relieve la existencia de un grupo reducido de participantes que son los que generaron el debate (comunity core), mientras el resto se limitaron a leer sus opiniones. En lo que se refiere a la participación de los lectores, los clubs de lectura virtuales no han de perseguir un número ilimitado de participantes, sino una conversación de calidad, una experiencia única en la que los lectores profundicen en la lectura, conversen serenamente, ocupen su tiempo de ocio en juegos literarios y en encuentros con otras personas que gustan, como ellos, de la alegría de leer y compartir.

Si bien son minoritarios los clubs de lectura en la universidad, consideramos necesario propiciar actividades que fomenten la lectura de ocio, también, en el entorno académico y, en esto, la biblioteca universitaria, corazón de la Universidad, tiene un papel esencial. 


\section{Referencias bibliográficas}

- Arana Palacios, J. (20I7). Clubes de lectura, en La lectura en España. Federación de Gremios de Editores de España. [Consulta: 25/oI/2OI7]

- Agustín Lacruz, M. del C., \& Moreno Gracia, E. (20I6). Los clubes de lectura en Aragón: Análisis descriptivo de una práctica socio-cultural de animación y promoción lectora]. Revista General de Información y Documentación, 26(2), 583-6o3.

https://doi.org/IO.5209/RGID.547I6

- Baldaquí, J.J. (20I8). Los clubs de lectura: entre la presencia y el mundo virtual. EN: LLuch, G. (ed) Claves para promocionar la lectura en la red. Madrid, Síntesis, I3O-I42.

- García-Rodríguez, A. y Gómez-Díaz, R. (2019). Plataformas y redes de lectura social. En Cordón-García, J. A. y Gómez-Díaz, R. (coords). Lectura, sociedad y redes: Colaboración, visibilidad y recomendación en el ecosistema del libro. Madrid: Marcial Pons, I4I-I86

- Gómez-Díaz, R., García-Rodríguez, A., Cordón-García, J. A. y Alonso-Arévalo, J. (20I6). Leyendo entre pantallas. Gijón: Trea.

- Jiménez Guerra, F. (2005). Clubes de lectura: una lectura oculta. Boletín GC Gestión Cultural, (I3), 3I. Retrieved from http://www.gestioncultural.org/boletin/pdf/bgci3-FJimenezGuerra.pdf

- Moreno-Mulas, M. A., García-Rodríguez, A., y Gómez-Díaz, R. (20I7). Conversando en la nube: cómo organizar un club de lectura virtual. Revista General de Información y Documentación, 27(I), I77-200. https://doi.org/IO.5209/RGID.56566.

- Salcedo, A. (2008). Club de Lectura Universidad de Murcia. Un recorrido por la lectura en sus seis años de vida. Educación y Biblioteca, 65, 89-93.

https://gredos.usal.es/jspui/bitstream/IO366/II9527/I/EB2O_Ni65_P89-93.pdf 